\title{
Protocol for an international, multicentre, prospective, observational study of nosocomial pneumonia in intensive care units: the PneumoINSPIRE study
}

Despoina Koulenti, Apostolos Armaganidis, Kostoula Arvaniti, Stijn Blot, Christian Brun-Buisson, Maria Deja, Jan De Waele, Bin Du, Joel M Dulhunty, Julia Garcia-Diaz, Matthew Judd, David L Paterson, Christian Putensen, Rosa Reina, Jordi Rello, Marcos I Restrepo, Jason A Roberts, Fredrik Sjovall, Jean-Francois Timsit, Sotirios Tsiodras, Jean-Ralph Zahar, Yuchi Zhang and Jeffrey Lipman, on behalf of the Working Group on Pneumonia of the European Society of Intensive

Care Medicine

Nosocomial pneumonia is a major problem in critical care settings. It is the most common health care-associated infection in intensive care units (ICUs) and the most common nosocomial infection contributing to death. ${ }^{1-4}$ In ICU settings, nosocomial pneumonia includes hospitalacquired pneumonia (HAP) that develops in non-intubated patients before or after admission to the ICU, and ventilator-associated pneumonia (VAP) in patients who require mechanical ventilation. HAP is defined as a lung infection in non-intubated patients arising $\geq 48$ hours after hospital admission. ${ }^{1,2}$ VAP is defined as pneumonia arising $\geq 48$ hours after endotracheal intubation. ${ }^{1,2}$ VAP represents about $80 \%$ of all episodes of nosocomial pneumonia in the ICU, and occurs in $9-27 \%$ of intubated patients. ${ }^{1-4}$

The reported incidence of nosocomial pneumonia varies widely, ranging from 3.1 to 18.6 cases per 1000 hospital admissions, with the presence of an artificial airway increasing the incidence by sixfold to 20-fold. ${ }^{1-6}$ This wide variation in incidence may be attributed to the lack of a gold standard for VAP diagnosis, and differences in definitions, study methods, patient populations and adherence to preventive measures. ${ }^{1-4,6-9}$ The incidence of VAP ranges from 1.9-3.8 cases per 1000 ventilator-days in the United States to $\geq 18$ cases per 1000 ventilator-days in Europe, and incidence increases with longer duration of mechanical ventilation. ${ }^{1-5}$ VAP accounts for more than half of all ICU antibiotic prescriptions and is associated with crude mortality rates of $20-71 \% .^{1-4}$ In addition, VAP is associated with an average increase in hospital length of stay of 7-9 days and an increase in health care costs of more than US $\$ 40000$ per patient. ${ }^{1}$ Studies of nosocomial pneumonia in non-intubated ICU patients are limited, with most information extrapolated from studies on VAP.1,3

The diagnosis of VAP is difficult as clinical criteria are subjective and non-specific; also, even for microbiologically confirmed VAP, there is significant interobserver variability. ${ }^{10-14}$ In addition, a poor correlation between

\begin{abstract}
Background: Nosocomial pneumonia in the critical care setting is associated with increased morbidity, significant crude mortality rates and high health care costs. Ventilatorassociated pneumonia represents about $80 \%$ of nosocomial pneumonia cases in intensive care units (ICUs). Wide variance in incidence of nosocomial pneumonia and diagnostic techniques used has been reported, while successful treatment remains complex and a matter of debate.

Objective: To describe the epidemiology, diagnostic strategies and treatment modalities for nosocomial pneumonia in contemporary ICU settings across multiple countries around the world.

Design, setting and patients: PneumoINSPIRE is a large, multinational, prospective cohort study of adult ICU patients diagnosed with nosocomial pneumonia. Participating ICUs from at least 20 countries will collect data on 10 or more consecutive ICU patients with nosocomial pneumonia. Sitespecific information, including hospital policies on antibiotic therapy, will be recorded along with patient-specific data. Variables that will be explored include: aetiology and antimicrobial resistance patterns, treatment-related parameters (including time to initiation of antibiotic therapy, and empirical antibiotic choice, dose and escalation or deescalation), pneumonia resolution, ICU and hospital mortality, and risk factors for unfavourable outcomes. The concordance of ventilator-associated pneumonia diagnosis with accepted definitions will also be assessed.
\end{abstract}

Results and conclusions: PneumoINSPIRE will provide valuable information on current diagnostic and management practices relating to ICU nosocomial pneumonia, and identify research priorities in the field.

Trial registration: ClinicalTrials.gov identifier NCT02793141.

Crit Care Resusc 2021; 23 (1): 59-66 


\section{ORIGINAL ARTICLES}

clinical interpretation and administrative surveillance methods for VAP has been reported in the US. ${ }^{15}$ Diagnosis of VAP and HAP is clinically important as false-positive results increase the risk of adverse events from unnecessary antibiotic treatment, contributing to the emergence of resistant organisms, while false-negative results may jeopardise patient survival through inadequate and delayed therapy. ${ }^{1-4,16-19}$

Successful treatment of nosocomial pneumonia is difficult and complex. ${ }^{1-3,20}$ Appropriate empiric antibiotic treatment, in terms of timeliness, dose and spectrum of cover, is a key element for effective management, while inappropriate treatment is associated with higher morbidity, mortality and costs. ${ }^{1-4,16-18}$ Up-to-date global data on everyday clinical practice regarding empiric and definitive therapeutic treatments, as well as de-escalation practices and their effects on patient outcomes, are essential for developing interventions aimed at improving and rationalising treatment choices. Exploration of the timeframes for resolution of nosocomial pneumonia, validation of previously identified predictors of adverse outcomes, and further investigation of new predictors of adverse outcomes will provide clinically significant information. ${ }^{21-31}$ Also, to our knowledge, few publications have addressed clinical characteristics and outcomes of nosocomial pneumonia in specific ICU subgroups, such as patients with chronic obstructive pulmonary disease (COPD) and older patients. ${ }^{32-37}$ Against this background, a large cohort study will improve our understanding of these factors and help identify optimised treatment approaches for specific subgroups of ICU patients. In this article, we describe the protocol for the PneumoINSPIRE study, which aims to address some of these gaps. PneumoINSPIRE has been registered with the ClinicalTrials.gov registry (identifier NCT02793141).

\section{Research objectives}

The aim of the PneumoINSPIRE study is to provide up-todate information on current clinical practice relating to ICU nosocomial pneumonia. The primary objectives are to: (i) evaluate the epidemiology of nosocomial pneumonia in ICU patients from multiple countries and regions, analysing responsible pathogens and resistance patterns by type of pneumonia and geographical region; (ii) describe, on a global scale, the current clinical practice regarding diagnosis of nosocomial pneumonia and determine the degree of adherence with current widely accepted definitions and guidelines; and (iii) identify variable treatment decisions for nosocomial pneumonia with emphasis on treatments, appropriateness, de-escalation practices and associated outcomes from a global perspective. The secondary objectives are to: (i) evaluate nosocomial pneumonia in specific subgroups of critically ill patients (ie, patients with COPD, older patients [aged $\geq 65$ years], and postoperative and trauma patients); (ii) describe the differences between nosocomial pneumonia that develops in non-intubated ICU patients and VAP; and (iii) compare the characteristics and outcomes between nosocomial pneumonia that develops in ward patients who are later transferred to an ICU and nosocomial pneumonia that arises in non-intubated ICU patients. The outcomes of interest are shown in Table 1.

\section{Study design and coordination}

PneumoINSPIRE is a prospective, international, multicentre, observational, prospective cohort study on nosocomial pneumonia in the ICU setting. The study logo is shown in Figure 1. The study was conceived and designed by the Working Group on Pneumonia of the European Society of Intensive Care Medicine (ESICM) and has received endorsement from the ESICM. The coordinating centre is the University of Queensland Centre for Clinical Research, located in Brisbane, Australia. Ethics approval was granted by the Royal Brisbane and Women's Hospital Human Research Ethics Committee (HREC/15/QRBW/35) and the Medical Research Ethics Committee of the University of Queensland (2015000302).

\section{Site selection}

Through investigator contacts and regional ICU network promotion, we aim to recruit at least 150 ICUs from at

\section{Table 1. Outcomes of interest}

- ICU and hospital mortality censored at Day 28

- Resolution of nosocomial pneumonia at Day 3, Day 7 and Day 14

- Recurrence of pneumonia at Day 14 and Day 28

- Concordance of clinical diagnosis of VAP with current widely accepted definitions (including the CDC definition of VAP) 1,2,38

- Mechanical ventilation-free days at Day 28 (censored at ICU discharge if discharged before Day 28)

- Antibiotic-free days at Day 28 (censored at ICU discharge if discharged before Day 28)

$C D C=$ Centers for Disease Control and Prevention. $I C U=$ intensive care unit. VAP = ventilator-associated pneumonia. 


\section{ORIGINAL ARTICLES}

Figure 1. PneumoINSPIRE study logo 19.

least 20 countries worldwide. There will be no limit set on the number of ICUs allowed to participate. All adult ICUs are eligible for participation in the PneumoINSPIRE study taking into consideration the following requirements: (i) ICUs agree to collect unit-related and patient-related data on site; (ii) ICUs agree to transfer the collected data to the coordinating centre; and (iii) ICUs pursue and obtain ethics committee approval or a waiver of ethics review.

\section{Participant recruitment}

Eligible patients are those meeting the inclusion criterion as diagnosed by the attending ICU clinician/s and none of the exclusion criteria (Table 2). Participating ICUs will collect data from a nominated start date until the minimum target of 10 consecutive ICU patients with an episode of nosocomial pneumonia has been reached or overall study recruitment has closed, whichever comes first. No maximum site targets have been set. The commencement of patient recruitment at a site level will vary and depend on local institutional ethics and site approval.

\section{Regional coordination}

Each participating country will have an appointed national coordinator, identified from among members of the Working Group on Pneumonia, members of national infection societies and highly esteemed intensivists in each country. As project leads, the national coordinators will facilitate site recruitment and have a key role in the conduct of the study for each participating country. The national coordinator will act as the primary contact for the coordinating centre and site principal investigators. This practice will help streamline data collection and problem solving. A local principal investigator will lead the study at each site (Supporting Information, Appendix 1).

\section{Data collection}

A data collection guide will be provided to each participating site in English or translated into one of the following languages: Spanish, French, Chinese, Portuguese and Italian. Research coordinators will enter all required data described in the protocol directly from source data to a paper-based and/or electronic case report form numbered with a consecutive site code and participant identification number. English, Spanish and Chinese versions of the case report form have been created. Non-identifiable study data will be collected and managed using the REDCap (Research Electronic Data Capture) tool, hosted at the University of Queensland. ${ }^{39}$ REDCap is a secure web-based application designed to support data capture for research studies. The coordinating centre will routinely monitor data entry in the database for completeness and consistency. A process of data validation - involving range, logic and missing data analysis - will be conducted before database lock. In cases where data clarification is required, the coordinating centre will send data queries to the site principal investigator. A site recruitment log that contains site recruitment details and participant identifiers will be kept by the site principal investigator in a secure and locked location. Data will only be re-identifiable by the site principal investigator. Site

\section{Table 2. Patient inclusion and exclusion criteria}

\begin{tabular}{ll}
\hline Inclusion criterion & Diagnosis with one of the following: \\
- & Ward HAP - admission to the ICU with a diagnosis of nosocomial \\
& pneumonia that developed in a non-intubated patient before ICU \\
& admission \\
- & ICU HAP — the first episode of ICU-acquired pneumonia that \\
& developed in a non-intubated patient \\
- & VAP — the first episode of ICU-acquired pneumonia in a patient \\
& receiving invasive ventilation for $\geq 48$ hours \\
- & Age $<18$ years \\
- & Nosocomial pneumonia in a patient receiving only palliative \\
& treatment at the time of assessment for eligibility (ie, the time of \\
& clinical diagnosis) \\
- & Previous inclusion in the study
\end{tabular}

$\mathrm{HAP}=$ hospital-acquired pneumonia. $\mathrm{ICU}=$ intensive care unit. VAP = ventilator-associated pneumonia. 


\section{ORIGINAL ARTICLES}

study materials will be kept for at least 5 years from study completion (ie, time of first publication) or as directed by local site approvals. The coordinating centre will retain study data for a minimum of 15 years from study completion.

\section{Site data}

Site-specific information will be collected once for each participating ICU (Table 3). Each ICU will record a limited dataset for all consecutive adult patients admitted to the ICU for the duration of the study - the total number of admissions during the study period and the total number of admissions of patients receiving mechanical ventilation for $\geq 48$ hours.

\section{Patient data}

For each patient with a clinical diagnosis of nosocomial pneumonia included in the study, detailed data will be obtained and entered into REDCap (Table 4). Supplementary material for the case report forms for nosocomial pneumonia with ICU onset and nosocomial pneumonia with onset outside the ICU is available online (Supporting Information, Appendix 2 and Appendix 3, respectively). As this study is observational, there will be no deviation from standard clinical practice and only data available from routine clinical practice will be recorded. Participating sites will not be required to perform specific laboratory or imaging tests if these are not part of their standard clinical practice.

\section{Sample size}

A sample size of 1000 ICU patients with nosocomial pneumonia is anticipated to comprise the dataset. As this study is primarily descriptive, no formal sample size calculations have been made. However, the sample size has been chosen to provide generalisable data that enables testing of about 10 variables in multivariable analysis, based on a minimum of 20 events per variable. ${ }^{41}$

\section{Statistical analysis}

Descriptive analytic techniques and parametric and non-parametric tests will be used to explore diagnosis, microbiological findings, subgroup differences and clinical outcomes. Cox regression will be used to predict dichotomous outcomes of interest, including mortality and resolution. Independent predictors and associated odds or hazard ratios, as appropriate, with 95\% confidence intervals will be reported. A two-sided $P$ value less than 0.05 will be considered statistically significant.

\section{Subgroup analyses}

Various subgroup analyses will be carried out, including by:

- type of nosocomial pneumonia (onset before ICU admission versus onset after ICU admission; in intubated versus non-intubated patients);

- geographic region;

- $\quad$ patients with COPD (yes versus no);

- age of patient (<65 years versus $\geq 65$ years);

- postoperative patients (yes versus no);

- trauma patients (yes versus no); and

- full concordance with accepted diagnostic criteria (yes versus no).

Concordance will be assessed by a specifically assigned review group consisting of the principal investigator and members of the steering committee or monitoring committee, with each recorded case of VAP assessed by two members of the group. In cases of disagreement, a third member will assess the case.

\section{Distribution of information}

Results will be presented in a timely manner at national and international conferences and in peer-reviewed journals.

\section{Summary}

PneumoINSPIRE is an international observational study on nosocomial pneumonia in ICU settings, including HAP in non-intubated patients and VAP. PneumoINSPIRE will describe the epidemiology, diagnostic strategies and treatment modalities for nosocomial pneumonia in geographically diverse ICUs. It will also explore implicated pathogens and their resistance patterns, resolution characteristics, prognostic factors and early indicators of patient outcomes. In addition, 


\section{ORIGINAL ARTICLES}

\section{Table 4. Patient-level data}

- Patient demographics: age, sex, race or ethnicity, weight, height, residence before hospital admission

- Presence of risk factors for colonisation with multidrug-resistant bacteria at hospital admission 1,2

- Date of hospital admission

- Diagnosis at hospital admission

- Date of ICU admission

- Location before ICU admission

- Type of ICU admission: medical, surgical or trauma; planned versus unplanned

- Diagnosis at ICU admission

- Severity of illness at ICU admission: APACHE II score, SAPS II, SOFA score

- Comorbidities and underlying diseases (including immunosuppression)

- Type of ventilation on ICU admission

- Date of initiation of NIMV and discontinuation of NIMV

- Date of intubation and extubation; location of intubation

- Date of initiation of discontinuation of invasive mechanical ventilation

- Presence of shock at ICU admission

- Presence of sepsis at ICU admission, including sepsis severity 40

- Date and time of nosocomial pneumonia onset (or suspicion of nosocomial pneumonia)

- Type of nosocomial pneumonia: onset before ICU admission versus onset after ICU admission; in intubated patients versus in nonintubated patients

- On day of pneumonia onset and ICU admission (for ward HAP): clinical signs and symptoms, laboratory data, radiological findings, sepsis severity, clinical severity assessment, CPIS, diagnostic procedures, date and time of diagnostic procedures, concurrent infection at a focus other than lungs

- Microbiological data, including identified pathogens and antibiogram where available

- Antibiotic management

- From Day 1 (day of onset for VAP or ICU HAP or day of ICU admission for ward HAP) to Day 14: clinical signs, severity of illness, laboratory results, diagnostic sampling results, and imaging if available; also, for VAP, respiratory data for up to 4 days before VAP onset

- On Day 3, Day 7 and Day 14: assessment of nosocomial pneumonia outcome as judged by the attending ICU physician (resolution, partial resolution, non-resolving or persistent)

- On Day 14 and Day 28: assessment for recurrence of nosocomial pneumonia or super-infection, or development of infection at another focus for the preceding period (ie, from Day 1)

- Vital status (censored at Day 28); coded as "alive" if discharged alive from the hospital before Day 28

APACHE $=$ Acute Physiology and Chronic Health Evaluation. $\mathrm{CPIS}=$ Clinical Pulmonary Infection Score. HAP $=$ hospital-acquired pneumonia ICU = intensive care unit. NIMV = non-invasive mechanical ventilation. SAPS = Simplified Acute Physiology Score. SOFA = Sequential Organ Failure Assessment. VAP = ventilator-associated pneumonia.

the study will examine how a diagnosis of VAP in routine clinical practice is associated with official definitions, and depict any discrepancies and their implications. We expect that the study findings will provide information that will be helpful for creating new clinical guidelines and identifying research priorities in the field of ICU nosocomial pneumonia.

Acknowledgements: We thank Sia Athanasas, Research Manager from the Burns, Trauma and Critical Care Research Centre, University of Queensland Centre for Clinical Research, for logistical support with this study. We also thank Simon Forsyth, Research Officer from the Health Information Systems Development Unit, Queensland Clinical Trials and Biostatistics Centre, School of Public Health, University of Queensland, for assistance with REDCap. Central coordination costs for the study have been partially funded by a research grant from the RBWH Foundation and a grant from the Rebecca L Cooper Medical Research Foundation. Despoina Koulenti (the coordinating principal investigator) was awarded a post-doctoral Early Career Researcher Fellowship from the University of Queensland and received an Ian Potter Travel Grant to attend a coordinating meeting for the study. 


\section{ORIGINAL ARTICLES}

\section{Competing interests}

Jan De Waele has received a grant from the Flanders Research Foundation (Senior Clinical Investigator Grant) and has consulted for Accelerate, Bayer HealthCare, Cubist, Grifols, MSD, Pfizer (honoraria were paid to his institution). Jeffrey Lipman has given lectures with honoraria from Pfizer and MSD. David Paterson has received research grants from AstraZeneca and has attended advisory boards for, acted as a consultant to, or given lectures with honoraria from Three Rivers Pharmaceuticals, Merck, AstraZeneca, Sanofi-Aventis, Pfizer, Johnson and Johnson, Shionogi and Leo Pharmaceuticals. All other authors declare no relevant competing interests.

\section{Author details}

Despoina Koulenti ${ }^{1,2}$

Apostolos Armaganidis 2

Kostoula Arvaniti ${ }^{3}$

Stijn Blot ${ }^{1,4}$

Christian Brun-Buisson ${ }^{5}$

Maria Deja6

Jan De Waele ${ }^{7}$

Bin $\mathrm{Du}^{8}$

Joel M Dulhunty 1,9,10

Julia Garcia-Diaz ${ }^{11,12}$

Matthew Judd ${ }^{9}$

David L Paterson ${ }^{1,13}$

Christian Putensen ${ }^{14}$

Rosa Reina ${ }^{15}$

Jordi Rello ${ }^{16,17,18}$

Marcos I Restrepo 19,20,21,22

Jason A Roberts 1,9,23,24

Fredrik Sjovall 25

Jean-Francois Timsit26,27

Sotirios Tsiodras ${ }^{28}$

Jean-Ralph Zahar 29,30

Yuchi Zhang ${ }^{31}$

Jeffrey Lipman 1,9,32

On behalf of the Working Group on Pneumonia of the European

Society of Intensive Care Medicine

1 University of Queensland Centre for Clinical Research (UQCCR), Faculty of Medicine, The University of Queensland, Brisbane, QLD, Australia.

2 Second Critical Care Department, Attikon University Hospital, Medical School, University of Athens, Athens, Greece.

3 Intensive Care Unit, Papageorgiou University Affiliated Hospital, Thessaloníki, Greece.

4 Department of Internal Medicine, Faculty of Medicine and Health Science, Ghent University, Ghent, Belgium.

5 Biostatistics, Biomathematics, Pharmacoepidemiology and Infectious Diseases Mixed Research Unit (French Institute for
Medical Research [INSERM], Université de Versailles Saint Quentin Medical School and Institut Pasteur), Paris-Saclay University, Montigny-Le-Bretonneux, France.

6 Lumbeck Klinik für Anästhesiologie und Intensivmedizin, Sektion Interdisziplinäre Operative Intensivmedizin, Universitatsklinikum Schleswig-Holstein, Campus Lübeck, Universität zu Lübeck, Lübeck, Germany.

7 Department of Critical Care Medicine, Ghent University Hospital, Ghent, Belgium.

8 Medical Intensive Care Unit, Peking Union Medical College Hospital, Beijing, China.

9 Department of Intensive Care Medicine, Royal Brisbane and Women's Hospital, Brisbane, QLD, Australia.

10 Research and Medical Education, Redcliffe Hospital, Brisbane, QLD, Australia.

11 Infectious Diseases Department, Ochsner Clinic Foundation, New Orleans, LA, USA.

12 Ochsner Clinical School, The University of Queensland, New Orleans, LA, USA.

13 Infectious Diseases Unit, Royal Brisbane and Women's Hospital,Brisbane, QLD, Australia

14 Department of Anesthesiology and Intensive Care Medicine, University Hospital Bonn, Bonn, Germany.

15 Critical Care Department, Hospital San Martin de la Plata, Buenos Aires, Argentina.

16 Clinical Research/Innovation in Pneumonia and Sepsis Research Group, Vall d'Hebron Research Institute, Barcelona, Spain.

17 Centro de Investigación Biomédica en Red en Efermedades Respiratorias, Instituto de Salud Carlos III, Madrid, Spain.

18 Clinical Research Department, Centre Hospitalier Universitaire de Nîmes, Nîmes, France.

19 Division of Pulmonary and Critical Care Medicine, Department of Medicine, University of Texas Health Science Center, San Antonio, TX, USA.

20 Pulmonary and Critical Care Fellowship Program, University of Texas Health Science Center, San Antonio, TX, USA.

21 Medical Intensive Care Unit, South Texas Veterans Health Care System, Audie L Murphy Division, San Antonio, TX, USA.

22 INnovation Science in Pulmonary Infections REsearch Network, Department of Medicine, University of Texas Health Science Center, San Antonio, TX, USA.

23 Centre for Translational Anti-infective Pharmacodynamics, School of Pharmacy, University of Queensland, Brisbane, QLD, Australia.

24 Pharmacy Department, Royal Brisbane and Women's Hospital, Brisbane, QLD, Australia.

25 Department of Intensive Care and Perioperative Medicine, Skane University Hospital, Malmö, Sweden.

26 Infection, Antimicrobials, Modelling, Evolution Research Centre, French Institute for Medical Research (INSERM), Université de Paris, Paris, France.

27 Medical and Infectious Diseases Intensive Care Unit (MI2), Hôpital Bichat, Assistance Publique - Hôpitaux de Paris, Paris, France. 


\section{ORIGINAL ARTICLES}

28 Fourth Department of Internal Medicine, Attikon University Hospital, Athens, Greece.

29 Service de Microbiologie Clinique et Unité de Contrôle et de Prévention du risque Infectieux, Groupe Hospitalier Paris Seine Saint-Denis, Assistance Publique - Hôpitaux de Paris, Bobigny, France.

30 Infection, Antimicrobials, Modelling, Evolution Research Centre, Unité Mixte de Recherche 1137, Université Paris 13, Sorbonne Paris Cité, Paris, France.

31 Department of Emergency Medicine, Tan Tock Seng Hospital, Singapore, Singapore.

32 Anesthesiology and Critical Care Department, Centre Hospitalier Universitaire de Nîmes, University of Montpellier, Nîmes, France.

Correspondence: d.koulenti@uq.edu.au

\section{References}

1 Kalil AC, Metersky ML, Klompas M, et al; American Thoracic Society and Infectious Diseases Society of America. Guidelines for the management of adults with hospitalacquired, ventilator-associated, and healthcare-associated pneumonia. Am J Respir Crit Care Med 2005; 171: 388-416.

2 Kalil AC, Metersky ML, Klompas M, et al. Executive summary: management of adults with hospital-acquired and ventilatorassociated pneumonia: 2016 clinical practice guidelines by the Infectious Diseases Society of America and the American Thoracic Society. Clin Infect Dis 2016; 63: 575-82.

3 Papazian L, Klompas M, Luyt CE. Ventilator-associated pneumonia in adults: a narrative review. Intensive Care Med 2020; 46: 888-906.

4 Rello J, Lisboa T, Koulenti D. Respiratory infections in patients undergoing mechanical ventilation. Lancet Respir Med 2014; 2: 764-74.

5 Koulenti D, Lisboa T, Brun-Buisson C, et al; EU-VAP/CAP Study Group. Spectrum of practice in the diagnosis of nosocomial pneumonia in patients requiring mechanical ventilation in European intensive care units. Crit Care Med 2009; 37: 2360-8.

6 Klompas M. What can we learn from international ventilatorassociated pneumonia rates? Crit Care Med 2012; 40: 3303-4.

7 Sousa AS, Ferrito C, Paiva JA. Application of a ventilator associated pneumonia prevention guideline and outcomes: a quasi-experimental study. Intensive Crit Care Nurs 2019; 51: 50-6.

8 Sousa AS, Ferrito C, Paiva JA. Intubation-associated pneumonia: an integrative review. Intensive Crit Care Nurs 2018; 44: 45-52.

9 Grgurich P, Hudcova J, Yuxiu L, et al. Diagnosis of ventilatorassociated pneumonia: controversies and working towards a gold standard. Curr Opin Infect Dis 2013; 26: 140-50.

10 Klompas M. Interobserver variability in ventilator-associated pneumonia surveillance. Am J Infect Control 2008; 36: 309-32.

11 Kallet $\mathrm{RH}$. The vexing problem of ventilator-associated pneumonia: observations on pathophysiology, public policy, and clinical science. Respir Care 2015; 60: 1495-1508.

12 Fernando SM, Tran A, Cheng W, et al. Diagnosis of ventilatorassociated pneumonia in critically ill adult patients - a systematic review and meta-analysis. Intensive Care Med 2020; 46: 1170-9.

13 Kollef $\mathrm{MH}$. Recognition of nosocomial pneumonia in the intensive care unit: still a confusing issue. Respir Care 2011; 56: 1209-12.

14 Nair GB, Niederman MS. Ventilator-associated pneumonia: present understanding and ongoing debates. Intensive Care Med 2015; 41: 34-48.

15 Skrupky LP, McConnell K, Dallas J, et al. A comparison of ventilator-associated pneumonia rates as identified according to the National Healthcare Safety Network and American College of Chest Physicians criteria. Crit Care Med 2012; 40: 281-4.

16 Iregui $M$, Ward S, Sherman G, et al. Clinical importance of delays in the initiation of appropriate antibiotic treatment for ventilator-associated pneumonia. Chest 2002; 1222: 262-268.

17 Kollef $\mathrm{MH}$, Sherman G, Ward S, Fraser VJ. Inadequate antimicrobial treatment of infections: a risk factor for hospital mortality among critically ill patients. Chest 1999; 115: 462-474.

18 Piskin N, Aydemir H, Oztoprak N, et al. Inadequate treatment of ventilator-associated and hospital-acquired pneumonia: risk factors and impact on outcomes. BMC Infect Dis 2012; 12: 268.

19 Luyt CE, Hékimian G, Koulenti D, Chastre J. Microbial cause of ICU-acquired pneumonia: hospital-acquired pneumonia versus ventilator-associated pneumonia. Curr Opin Crit Care 2018; 24: 332-8.

20 Torres A, Niederman MS, Chastre J, et al. International ERS/ ESICM/ESCMID/ALAT guidelines for the management of hospital-acquired pneumonia and ventilator-associated pneumonia: guidelines for the management of hospitalacquired pneumonia (HAP)/ventilator-associated pneumonia (VAP) of the European Respiratory Society (ERS), European Society of Intensive Care Medicine (ESICM), European Society of Clinical Microbiology and Infectious Diseases (ESCMID) and Asociación Latinoamericana del Tórax (ALAT). Eur Respir J 2017; 50: 1700582.

21 Vidaur L, Gualis B, Rodriguez A, et al. Clinical resolution in patients with suspicion of ventilator-associated pneumonia: a cohort study comparing patients with and without acute respiratory distress syndrome. Crit Care Med 2005; 33: 1248-53.

22 Dennesen PJ, van der Ven AJ, Kessels AJ, et al. Resolution of infectious parameters after antimicrobial therapy in patients with ventilator-associated pneumonia. Am J Respir Crit Care Med 2001; 163: 1371-5. 


\section{ORIGINAL ARTICLES}

23 Luna CM, Blanzaco D, Niederman MS, et al. Resolution of ventilator-associated pneumonia: prospective evaluation of the clinical pulmonary infection score as an early clinical predictor outcome. Crit Care Med 2003; 31: 676-82.

24 Kollef KE, Schramm GE, Wills AR, et al. Predictors of 30day mortality and hospital costs in patients with ventilatorassociated pneumonia attributed to potentially antibioticresistant gram-negative bacteria. Chest 2008; 134: 281-7.

25 Esperatti M, Ferrer M, Giunta $V$, et al. Validation of predictors of adverse outcomes in hospital-acquired pneumonia in the ICU. Crit Care Med 2013; 41: 2151-61.

26 Combes A, Luyt CE, Fagon JY, et al; for the PNEUMA Trial Group. Early predictors for infection recurrence and death in patients with ventilator-associated pneumonia. Crit Care Med 2007; 35: 146-54.

27 Pena C, Gomez-Zorilla S, Oriol I, et al. Impact of multidrug resistance on ventilator-associated Pseudomonas aeruginosa outcome: predictors of early and crude mortality. Eur J Clin Microbiol Infect Dis 2013; 32: 413-20.

28 loannas $M$, Ferrer $M$, Cavalcanti $M$, et al. Causes and predictors of nonresponse to treatment of intensive-care unit acquired pneumonia. Crit Care Med 2004; 32: 938-45.

29 Leroy O, Meybeck A, d' Escrivan T, et al. Hospital-acquired pneumonia in critically ill patients: mortality risk stratification upon onset. Treat Respir Med 2004; 3: 123-31.

30 Bowton DL. Resolution of ventilator-associated pneumonia: is my patient getting better? Crit Care Med 2010; 38: 308-9.

31 Vidaur L, Planas K, Sierra R, et al. Ventilator-associated pneumonia: impact of organisms on clinical resolution and medical resources utilization. Chest 2008; 133: 625-32.

32 Nseir S, Di Pompeo C, Soubrier S, et al. Impact of ventilatorassociated pneumonia on outcome in patients with COPD.
Chest 2005; 128: 1650-6.

33 Makris D, Desrousseaux B, Zakynthinos E, et al. The impact of COPD on ICU mortality in patients with ventilator-associated pneumonia. Respir Med 2011; 105: 1022-9.

34 Koulenti D, Blot S, Dulhunty J, et al; and the EU-VAP/CAP Study Group. COPD patients with ventilator-associated pneumonia: implications for management. Eur J Clin Microbiol Infect Dis 2015; 34: 2403-11.

35 Koulenti $D$, Parisella $F R$, Xu E, et al. The relationship between ventilator-associated pneumonia and chronic obstructive pulmonary disease: what is the current evidence? Eur J Clin Microbiol Infect Dis 2019; 38: 637-47.

36 Blot S, Koulenti D, Dimopoulos, et al; and the EU-VAP/CAP Study Group. Incidence, risk factors, and mortality for ventilator-associated pneumonia in middle-aged, old, and very-old critically ill patients. Crit Care Med 2014; 42: 601-9

37 Koulenti D, Tsigou E, Rello J. Nosocomial pneumonia in 27 ICUs in Europe: perspectives from the EU-VAP/CAP study. Eur J Clin Microbiol Infect Dis 2017; 36: 1999-2006.

38 Magill SS, Klompas M, Balk R, et al. Developing a new, national approach to surveillance for ventilator-associated events. Crit Care Med 2013; 41: 2467-75.

39 Harris PA, Taylor R, Thielke R, et al. Research electronic data capture (REDCap) - a metadata-driven methodology and workflow process for providing translational research informatics support. J Biomed Inform 2009; 42: 377-81.

40 Singer $M$, Deutschman CS, Seymour CW, et al. The third international consensus definitions for sepsis and septic shock (Sepsis-3). JAMA 2016; 315: 801-10.

41 Ogundimu EO, Altman DG, Collins GS. Adequate sample size for developing prediction models is not simply related to events per variable. J Clin Epidemiol 2016; 76: 175-182. 